\title{
Congenital amniotic band syndrome: Report of a familial recurrence
}

\author{
W. LEE IRVING, DO \\ GARY L. DOUBLESTEIN, DO
}

A case of familial congenital amniotic band syndrome is reported. The infant had deformities of the fingers of both hands, anencephaly, and a missing left testis. The mother had a reduction deformity of the right fingers and thumb. This is thought to be only the second case of mother-son involvement; most other familial recurrence involves more distant relatives. A review of etiologic theories shows that amniotic rupture and subsequent band formation has been the dominant theory, but the implications of the few familial cases, malformations not associated with bands, and experimental data have raised some doubts about the primary mechanism.

Amniotic band syndrome generally occurs in sporadic fashion. A few familial cases have been reported, however. In our case, the mother had a deformity of the right hand as a result of amniotic band. The fetus had severe band-associated deformities, including anencephaly and a missing left testis.

\section{Report of case}

A 19-year-old woman (gravida 1, para O) presented to the clinic in her 20 th week of gestation. She had an unremarkable past medical history, except for a deformity of the right hand since birth. The deformity left her with no fingers and only a thumb remnant (Fig 1).

Otherwise, the patient was well developed. Fundal height was consistent with gestational age, and fetal heart rate was 140 beats per minute. Blood pressure was $120 / 180 \mathrm{~mm} \mathrm{Hg}$. Routine screening revealed a positive
Chlamydia culture, for which she was placed on erythromycin therapy and given appropriate medical advice, including an appointment for repeat culture. However, the patient did not return to the clinic until two months later. Examination results were essentially normal and consistent with dates. The patient had not taken her medication, so she was warned about noncompliance and about not keeping appointments, and instructed about the importance of prenatal care.

In spite of numerous follow-up attempts, however, she was not seen in the clinic until her 36th week of gestation. At that point, the fundal height had lagged behind gestational age by about three weeks. A culture again was positive for Chlamydia. Leopold maneuvers indicated that the fetus was in breech presentation. However, the fetal head could not be palpated. Ultrasonography confirmed breech presentation but no discernible calvarium. Normal corpus callosum was present, but ventricular structures were not found. Oligohydramnios also was noted (Fig 2).

An abdominal roentgenographic examination confirmed the failure of calvarium development, with a rudimentary occipital bone. Fetal arms and legs were splayed, with the hands positioned near the head on either side (Fig 3).

The patient and her family were informed that the fetus was anencephalic and that the survival expectancy would be extremely low. She was also advised that the ultimate demise of the fetus precluded the maternal risk of cesarean section. Chlamydia treatment was restarted.

An amniotomy was performed. Pitocin induction was successful and after complete cervical dilation and administration of spinal anesthesia, a Pinard maneuver was performed for the assisted breech delivery.

The infant boy was severely deformed. Instead of true anencephaly, however, the deformities were caused by amniotic bands that prevented formation of the calvarium, nose, eyes, and maxilla. Both hands had been trapped in the bands, with deformities of the fingers as a result. Soft, diffluent brain tissue was present but encased in a thin, transparent membrane (Fig 4). The left 


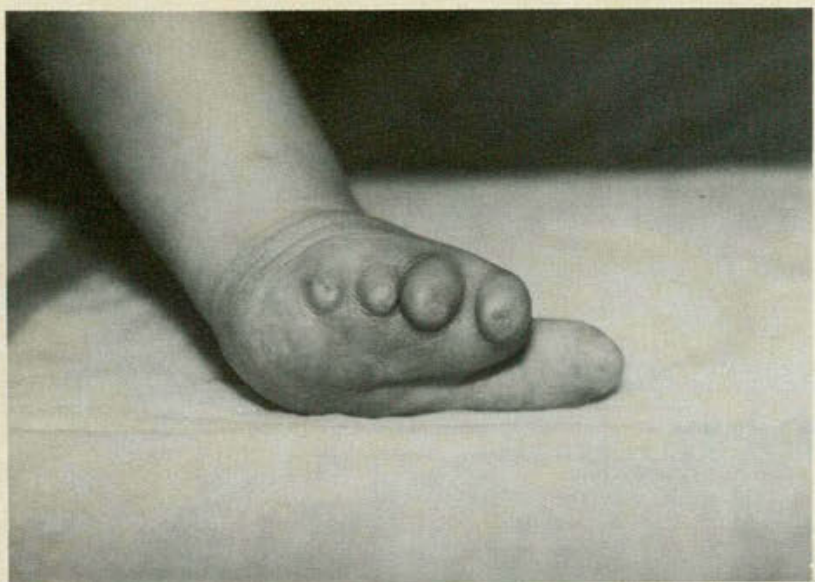

Fig 1. Mother of anencephalic fetus. Note that fingers appear to be "pinched off," indicating amniotic band in utero.

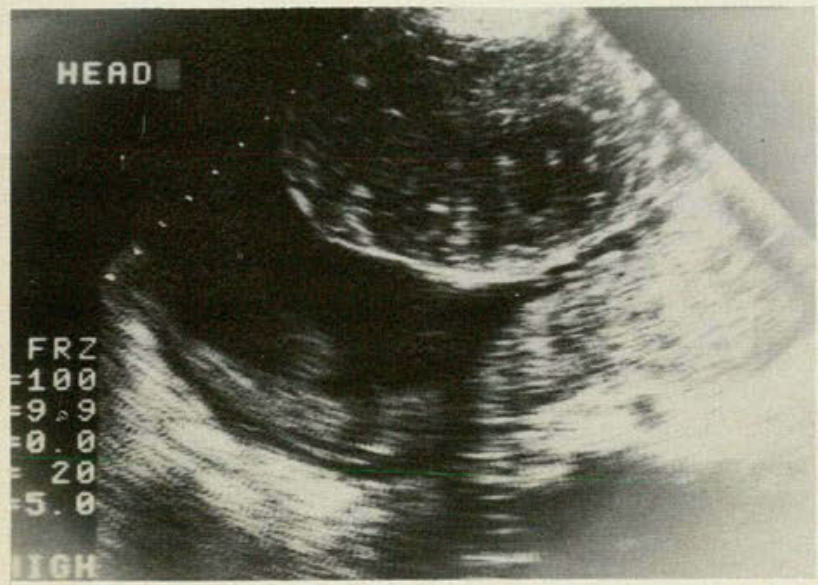

Fig 2. Absence of calvarium. Note normal corpus callosum but absence of ventricular structures.

testis was absent.

The infant was given whiffs of oxygen and kept warm. Although there were initial spontaneous respirations, he died within one hour after birth.

The mother had an unremarkable postpartum course and was discharged in satisfactory condition. She kept her follow-up appointments in the clinic. On Sept 9, 1986, she returned to the clinic at about 12 weeks' gestation. At 27 weeks' gestation, high-resolution ultrasonographic examination and genetic studies indicated a healthy fetus with no evidence of deformity. She gave birth to a healthy infant girl free of malformations.

\section{Discussion}

The incidence of amniotic band syndrome ranges from $1: 1,200$ to $1: 15,000$ deliveries. ${ }^{1,2}$

Review of the literature showed only rare reports of familial occurrences of amniotic band syndrome; most of these involved rather distant relations..$^{3,4}$ To our knowledge, only one other case of familial congenital amniotic band syndrome has been reported directly from mother to fetus. ${ }^{5}$

The prevailing view has been that there is no genetic predisposition or recurrence rate with amniotic band syndrome. ${ }^{2,6}$ However, if this paper is followed by other reports of familial occurrence, perhaps this opinion will have to be reassessed.

Various theories have been presented to explain the etiology of the amniotic band syndrome. ${ }^{7,8,9}$ Torpin $^{9}$ observed that amniotic bands derive from early rupture of the amnion which forms mesodermal strings and naked chorion. By abrasion and constriction of these bands, abnormalities of the fetus are created. Thus there have been reports of amniotic band syndrome with chorionic villi sample and amniocentesis.

However, Hunter and Carpenter ${ }^{10}$ reported on four cases that were inexplicable by Torpin's theory. They raise the question that there is the possibility that the amniotic band may be a manifestation of an underlying fetal defect that might result from extrinsic or intrinsic causes. Our presentation would agree with the suggestion, since anencephaly could very well have been the initiating event with subsequent formation with amniotic bands.

Defects of the extremities are the most common anomalies in this syndrome. They include constriction rings or amputations of digits or limbs, pseudosyndactyly, clubbed feet, or abnormal patterns of the dermal ridges. Craniofacial deformities, including asymmetric encephalocele, anencephaly, asymmetric microphthalmia, facial clefting, and nasal deformity, are the next most common. Visceral manifestations, such as gastroschisis, omphalocele, bladder exstrophy, and vertebral hypoplasia, occur less often. Internal organ deformities are rare. Up to $77 \%$ of cases involve anomalies. ${ }^{1}$

Pregnancies that ordinarily show no special characteristics result in delivery of infants with amniotic band syndrome. Malpresentation, a feature in the case reported here, is not more common than usual. However, some reports suggest an increased abortion and prematurity rate. ${ }^{1}$

There are currently no prenatal tests to identify affected fetuses, with the exception of real-time ultrasonography. This examination looks for an aberrant sheet of amniotic membrane floating freely near the fetus. However, one must also identify a fetal anomaly before electing to terminate the pregnancy, because a free floating edge of amniotic membrane without an associated fetal deformity is not sufficient for diagnosis. ${ }^{2}$ Herbert and co-workers ${ }^{7}$ 


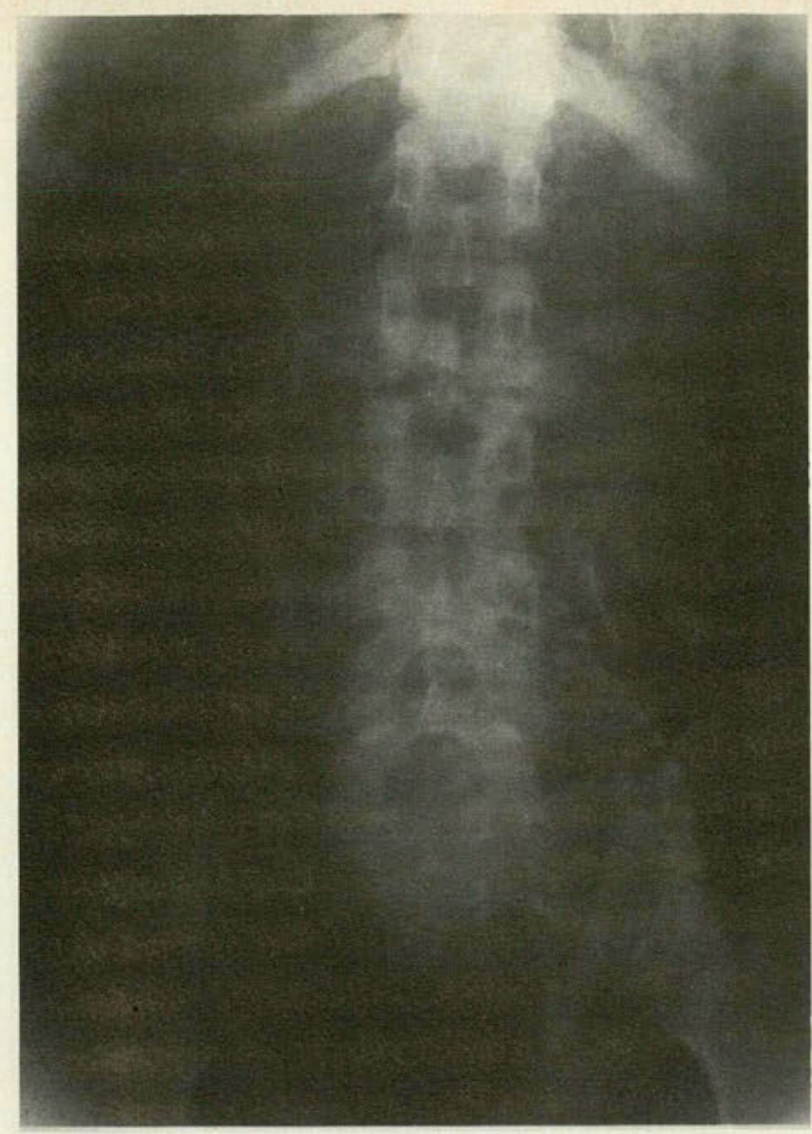

Fig 3. Flat abdominal $x$-ray film at 36 weeks' gestation showing splayed configuration of fetal limbs and absence of skull formation.

recommend serial ultrasonographic examinations to exclude causes of intrauterine membrane and look for associated malformations.

A-fetoprotein (AFP) screening may be of help prenatally if the amniotic band has caused a defect in the neural tube. ${ }^{11}$

The literature suggests that cases of amniotic band syndrome with severe craniofacial deformities are seldom correctly diagnosed at birth. Amniotic band syndrome may be present in as many as $5 \%$ of anencephalic infants. ${ }^{1}$ The diagnosis is not amenable to genetic evaluation, because the karyotype is usually normal.

Amniotic band syndrome should be suspected with asymmetric defects that do not occur along normal embryologic lines or by the presence of fibrous band encircling limbs, fingers, or toes. ${ }^{2}$

\section{Comment}

Prenatal diagnosis of amniotic band syndrome has met with many difficulties, but careful ultrasonogra-

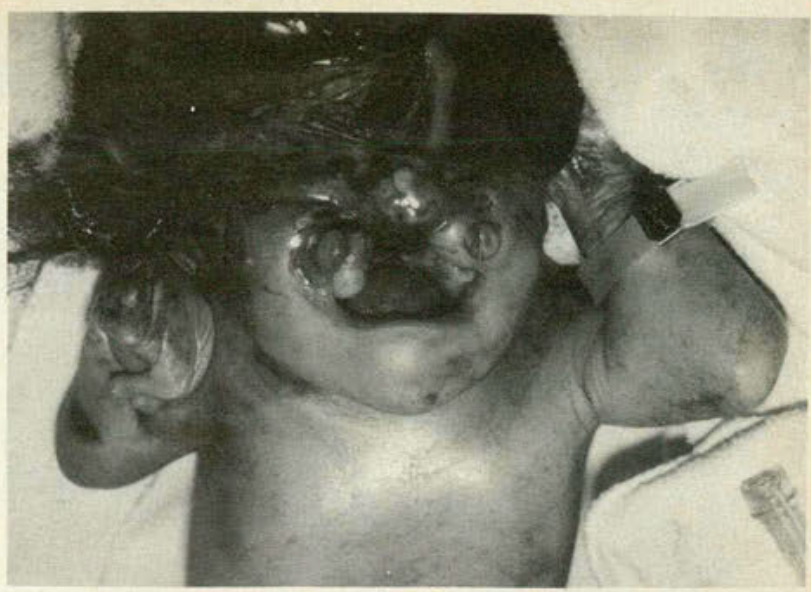

Fig 4. Fetus with anencephaly secondary to amniotic band. Note deformities of hands entrapped in band. Brain tissue is present but encased in transparent membrane. Maxilla, nose, eyes, and skull are absent.

phic diagnosis shows promise. AFP screening may result in more frequent prenatal diagnoses of the anencephalic fetus if it becomes the standard of prenatal care. Careful evaluation at the time of delivery must be done to distinguish this syndrome from typical neural tube defects, chromosomal anomaly, or cleft deformities.

1. Seeds JW, Cefalo RC, Herbert WP: Amniotic band syndrome. Am J Obstet Gynecol 1982;144:243-248.

2. Mahony BS, Filly RA, Callen PW, et al: The amniotic band syndrome: Antenatal sonographic diagnosis and potential pitfalls. Am J Obstet Gy. necol 1985; 152:63-68.

3. Lubinsky M, Sujansky E, Sanger W, et al: Familial amniotic bands. Am J Med Genet 1983;14:81-87.

4. Lubinsky M: Familial amniotic bands, letter. J Pediatr 1983;102:323.

5. Etches FC, Stewart AR, Ives EJ: Familial congenital amputations. $J$ Pediatr 1982;101:448-449.

6. Rushton DI: Amniotic band syndrome. Br Med $J$ 1983;286:919-920.

7. Herbert WN, Seeds JW, Cefalo RC: Prenatal detection of intraamniotic bands: Implications and management. Obstet Gynecol 1985;65 (Suppl 3):36S-38S.

8. Planteydt HT, Vandevooren MJ, Verweij J, et al: Amniotic band malformations in a child born after pregnancy screened by chorionic villus biopsy. Lancet 1986;2:756-757.

9. Torpin R: Fetal Malformation, Springfield, IIl, Charles C Thomas, Publisher, 1986 pp 3-137.

10. Hunter AGW, Carpenter DF: Implications of malformations not due to amniotic band sequence. Am J Med Genet 1986;24:691-700.

11. Maeri JN, Baker DA, Baim RS: Diagnosis of neural tube defects by evaluation of amniotic fluid. Clin Obstet Gynecol 1981;24:1089-1102.

From the Department of Obstetrics and Gynecology, Metropoli$\tan$ Hospital, Grand Rapids, Mich.

For reprints, address Dr Irving, 141-2 Delta Dr, Minot AFB, ND 58704. 
